\title{
Spectrum-Efficient Cognitive Radio Transceiver Using Multiwavelet Filters
}

\author{
Manju Mathew, A. B. Premkumar, and A. S. Madhukumar \\ Centre for Multimedia and Network Technology, School of Computer Engineering, Nanyang Technological University, \\ 50 Nanyang Avenue, Singapore 639798 \\ Correspondence should be addressed to Manju Mathew, manj0001@ntu.edu.sg
}

Received 31 March 2012; Accepted 31 May 2012

Academic Editors: H. Ikeda and C. Yang

Copyright () 2012 Manju Mathew et al. This is an open access article distributed under the Creative Commons Attribution License, which permits unrestricted use, distribution, and reproduction in any medium, provided the original work is properly cited.

Cognitive radio (CR) transceiver that can offer adequate data rate and multiuser support for future wireless networks is a promising technology for reliable and spectrum-efficient mobile communication. Orthogonal frequency division multiplexing (OFDM) and scalar wavelet based schemes have been proposed as physical layer techniques for CR. This paper proposes multiwavelet packetbased multicarrier multiple-access scheme as an equally promising candidate for multi-user CR networks and using existing orthonormal multiwavelets, the performance of the proposed system is evaluated. It is shown that the error performance of the proposed system under frequency and phase offset conditions is comparable with existing schemes.

\section{Introduction}

With the ability to learn from and adapt to both radio environment and user needs, CR promotes viable communication and optimizes the use of radio frequency (RF) spectrum [1]. Even though OFDM-based systems have been proposed to be the most promising candidate for $\mathrm{CR}$ transmission [2-6], recent research work propose waveletbased schemes to be an alternative modulation technique in terms of flexibility, adaptivity, and spectrum efficiency [7]. The efficacy of scalar wavelet packets for CR-based systems has been explored, and various results are discussed in [813]. Both analytical and experimental results show that scalar wavelet-based schemes insures better flexibility, sidelobe suppression, and reconfigurability at moderate complexity. Wavelet theory has been enriched by the introduction of multiwavelets, and it has been proved that they incorporate more degrees of freedom and additional advantages than scalar wavelets [14]. Hence the vector extension of scalar wavelet packet called multiwavelet packet is explored in this work for multi-user CR applications. Multiwavelet packetbased multi carrier multiple-access scheme (MWP-MC-MA) for $\mathrm{CR}$ applications is proposed for the first time in the literature. The uplink and downlink system model and signal model are described in detail, and necessary equations are discussed.

The rest of the paper is organized as follows. In Section 2 a brief review on multiwavelets is given, and its communication-based applications available in the literature are highlighted. Proposed spectrum sensing method is outlined in Section 3. The system model and signal model of single user and multi-user CR environment are described in Sections 4 and 5 . The performance analysis of the proposed system is portrayed in Section 6. Conclusions and future directions are given in Section 7.

\section{A Brief Review on Multiwavelets and Their Advantages}

To clearly investigate the characteristics of multiwavelets and to emphasize the motivation behind this proposal, a brief review on multiwavelets and their unique advantages is described in this section.

2.1. Multiwavelets and Multifilters. Multiwavelets are the generalization of multiresolution analysis (MRA) [14]. The two-scale relations lead to scaling functions and wavelet 
functions similar to that in scalar wavelets. But the equations are two-scale matrix equations and can be given as

$$
\begin{aligned}
& \Phi(t)=\sum_{n} H(n) \Phi(2 t-n), \\
& \Psi(t)=\sum_{n} G(n) \Psi(2 t-n),
\end{aligned}
$$

where $\Phi(t)=\left[\phi_{1}(t) \phi_{2}(t) \cdots \phi_{r}(t)\right]^{T}$ and $\Psi(t)=\left[\psi_{1}(t)\right.$ $\left.\psi_{2}(t) \cdots \psi_{r}(t)\right]^{T}$ form the set of scaling functions and corresponding wavelets. The suffix $r$ denotes the number of wavelets and is dubbed as multiplicity. Each $H(n)$ and $G(n)$ are square matrices of size $r \times r$ and are termed as multifilters [14]. The matrix structure of the filter bank provides more degrees of freedom for multiwavelets. Unlike scalar wavelets, multiwavelet system can simultaneously provide perfect reconstruction while preserving length (orthogonality), good performance at the boundaries (linear-phase symmetry), and a high order of approximation (vanishing moments) together with short support [15]. Hence superior performance is expected from multiwavelets in signal and image processing applications compared to scalar wavelets. To exploit the communication aspects of multiwavelets for multicarrier modulation, it is required to prove the existence of orthogonal multiwavelet packet subspaces and this is discussed in [16]. Unlike scalar wavelets, high-pass filter coefficients of the multifilter cannot be obtained by alternating flip of the low-pass filter but have to be designed [17]. Design of various orthogonal multifilter banks is described in [18-21]. Application of multiwavelet packets for digital communications was first proposed by You and Ilow [22]. They have proposed the vector extension of Mallat's algorithm to implement multiwavelet packet modulation (MWPM) and have proved that spectrum efficiency will be increased $r$ times compared to OFDM and scalar WPM. Besides, additional results are presented in [23] to verify the adaptability of MWPM to mitigate strong narrow-band interference with its flexible time frequency tiling. The symbol-overlapped multiwavelet OFDM for system capacity improvement is discussed in [24]. A simpler method to obtain direct and inverse multiwavelet transform using toeplitz matrix formation is described in [25]. All the research results prove that multifilter banks can bring enormous advantages in next-generation wireless networks. But the use of multiwavelet filter banks to implement a spectrumefficient and adaptive modulation scheme within CR context is still to be explored. The multiple-access capability of MWPM has not been addressed so far in the literature. Performance evaluation of multiwavelets in the literature is limited to the filter banks proposed in $[18,19]$, and hence a fair comparison among different orthogonal multiwavelets in terms of error performance is yet unavailable. These issues are tried to address in the following sections and are the main contributions of this work.

2.2. Advantages of MWPM for CR. Features of orthonormal multiwavelet packets that make them suitable for CR applications are listed below. (i) In the case of multiwavelets, each filter coefficient is an $r \times r$ matrix, and hence larger number of subcarriers are possible for a given bandwidth. For a transform size of $N$ and multiplicity $r$, there can be as many as $r 2^{N}$ orthogonal subbands and hence $r$ times bandwidth efficiency. This helps allocating more subbands among different users compared to scalar wavelets and OFDM.

(ii) The discrete multiwavelet packet transform (DMWPT) implemented for data reception can be used for spectrum sensing at no additional cost. As multiwavelets provide better results in feature extraction and signal denoising compared to scalar wavelets, the multiwavelet spectrum estimator can outperform scalar wavelet-based spectrum detector described in [26].

(iii) Multiwavelets maintain orthogonality even with certain integer shifts. If the support length of multiwavelet base is $(0, L)$, the orthogonality condition is satisfied for integer shifts of the wavelet base up to $L$. These shifted waveforms can be used to modulate different data of the same user. Thus the capacity of the system can be improved $L$ times for a given transform size, or the number of subcarriers can be reduced for a given data rate [24]. Thus multiwaveletbased system offers high degree of flexibility and adaptivity in terms of data rate, modulation, and number of subcarriers. OFDM subcarriers overlap only in frequency domain, and hence this kind of flexibility cannot be achieved in OFDM-based systems.

\section{Spectrum Sensing with Multifilters}

Spectrum sensing is the major task of $\mathrm{CR}$ as it is restricted to operate in unused bands. This is not common in any conventional system and hence its successful implementation is a crucial task [27]. Considerable research has been done in this area and numerous algorithms and architectures are available in literature [26-37]. The existing research results prove that spectrum sensing can be implemented in two stages-preliminary or coarse sensing at the physical layer and fine sensing at the MAC layer [28]. Energy or power detector is suggested as the preliminary sensing technique as its algorithm does not require prior knowledge of the signal characteristics. In this work, multifilter banks are exploited to calculate energy of the given spectrum and thus to locate the vacant bands. The related work, motivation, and the proposed sensing algorithm are given in the subsequent subsections.

3.1. Related Work and Motivation. Locating the vacant bands using filter banks is already addressed in the literature [29], and the merits and demerits are discussed. It has been shown that if the filter banks that can be employed for multicarrier communication of CR networks are utilized for spectrum sensing, the sensing circuit can be implemented at no additional cost [29]. The detailed performance analysis of various 
filter-bank-based spectrum sensing is presented in [29]. Wavelet filter bank-based-sensing is discussed in $[26,30]$. A wavelet packet-based energy detector using infinite Impulse Response (IIR) scalar wavelet filters is described in [30]. IIR filters are used for sensing purpose to reduce the complexity of the sensing circuit. But this demands additional circuitry in the CR transceiver for sensing the spectrum. The use of finite impulse response (FIR) wavelet filter bank for spectrum sensing is outlined in [26] and it has been shown that the results are comparable with existing power estimation techniques. Since the sensing circuit proposed in [26], uses scalar wavelet FIR filters, its implementation is possible by utilizing wavelet-based CR receiver (demodulator) at no additional hardware circuitry. Multiwavelet FIR filter-bankbased spectrum sensing is proposed in this section which has not been addressed in the previous works. The motivation behind this proposal is the added advantage of multifilterbased spectrum sensing compared to existing filter bankbased-sensing and is listed below.

(i) Reliable feature extraction. In multiwavelet filter banks, each filter coefficient is a square matrix of size $r \times r$. Hence the filtering operation using multiwavelets involves matrix convolution. This demands $r$ input rows in multifilter based operations. The additional input rows are obtained by repeated row preprocessing which increases the reliability of feature extraction [14].

(ii) Lower number of iterations. Even though each step of multiwavelet transform is computationally complex than scalar wavelet transform, the required frequency (time) resolution can be achieved with reduced number of iterations (at lower transform size). Hence the overall complexity of multiwavelet transform will be equivalent to scalar wavelet transform. That is, for multiwavelets of multiplicity $r$. Each iteration generates $r \cdot 2^{j}$ subbands where $j$ is the iteration level. If $r$ is a power of $2,(N-a)$ iterations are sufficient to generate $2^{N}$ subbands where $r=2^{a}$.

(iii) Lower number of filter taps. According to [30] the complexity of wavelet filter-bank-based spectrum sensing is $L \cdot N \log _{2} N$ where $L$ is the filter length and $N$ is the transform size. Multiwavelet FIR filters have lower number of filter coefficients compared to scalar FIR filters within the same support length. Hence the complexity of sensing is comparable to that of IIR filter-based spectrum sensing and is lower than that of FIR filter-based scalar wavelet spectrum sensing.

3.2. Multifilter-Based Sensing Methodology and Algorithm. The spectrum analysis and data transmission proposed in this work are limited to multifilter banks of multiplicity two. Since multiplicity is two, spectrum sensing circuit needs two input rows which can be easily obtained by single repetition of the signal stream under consideration. Generating the multiwavelet coefficients of the signal stream is shown in Figure 1 . The input stream is repeated, and the $2 \times 2 \mathrm{ma}-$ trix filters operate on both streams to generate four output

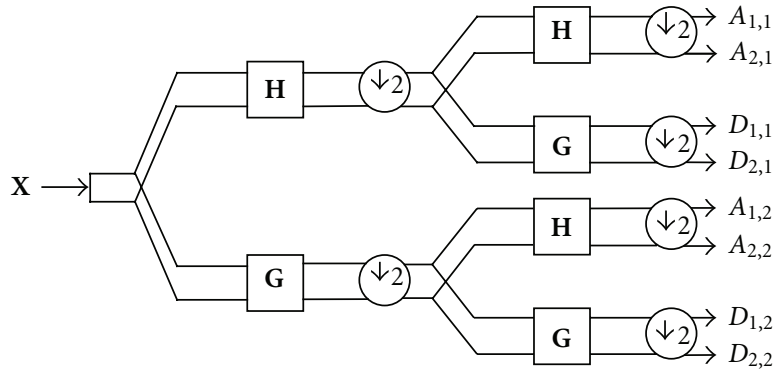

Figure 1: Signal decomposition using multifilters.

streams which are downsampled by a factor of 2 . The decomposition process is repeated until the desired level of frequency-time resolution is achieved. Each row of the multifilter is a combination of two ordinary filters, one operating on the first data stream and the other operating on the second stream. For a given transform size, the output of the DMWPT will contain $2 \cdot 2^{N}$ multiwavelet packet coefficients. It is shown that at level two iteration, there are eight wavelet packet coefficients or eight frequency subbands. The coefficients corresponding to low-pass filtering and downsampling are termed as $A_{i, j}$ where $A$ denotes approximation, $i$ represents corresponding row, and $j$ represents the continuous array sequence. Similarly, high-pass filtered coefficients are represented as $D_{i, j}$. Successive iterations of filtering and downsampling can also be obtained using toeplitz matrix [14]. Let $\mathbf{H}[\mathbf{0}], \mathbf{H}[\mathbf{1}]$, and $\mathbf{H}[2]$ be the lowpass filter coefficients. The doubly infinite toeplitz matrix corresponding to low-pass filter will be

$$
\left[\begin{array}{cccccccc}
\mathrm{H}[2] & \mathrm{H}[\mathbf{1}] & \mathrm{H}[\mathbf{0}] & \mathbf{0} & \mathbf{0} & \mathbf{0} & \mathbf{0} & \cdots \\
\mathbf{0} & \mathbf{0} & \mathrm{H}[2] & \mathrm{H}[\mathbf{1}] & \mathrm{H}[\mathbf{0}] & \mathbf{0} & \mathbf{0} & \cdots \\
\vdots & \vdots & \vdots & \vdots & \vdots & \vdots & \vdots & \ddots \\
\mathbf{0} & \mathbf{0} & \mathbf{0} & \mathbf{0} & \cdots & \mathrm{H}[2] & \mathrm{H}[\mathbf{1}] & \mathrm{H}[\mathbf{0}]
\end{array}\right],
$$

where each element in the matrix is a $2 \times 2$ matrix. In practical, the size of the toeplitz matrix depends on the length of the signal sequence under consideration. In a similar manner, the toeplitz matrix can be obtained for high-pass filter. Thus the calculation of wavelet packet coefficients in each level of the signal stream can be done by simple matrix multiplication and is given as

$$
\mathbf{C}_{L}^{(j)}=\mathbf{T}_{L}^{(j)} \mathbf{X}^{(j)},
$$

where $\mathbf{C}_{L}^{(j)}$ are the multiwavelet coefficients, $\mathbf{T}_{L}^{(j)}$ is the low pass filter toeplitz matrix, and $\mathbf{X}^{(j)}$ is the input vector at level $j$. It is important to note that each element of $\mathbf{C}_{L}^{(j)}$ and $\mathbf{X}$ is a vector instead of scalar with size $2 \times 1$. Hence $\mathbf{C}_{L}^{(j)}$ corresponds to coefficient value of two subbands. Similarly $\mathbf{C}_{H}^{(j)}$ can also be calculated corresponding to high-pass filter and thus completes the process of obtaining coefficients of level $j$. Once the multiwavelet packet coefficients are obtained, the energy contained in a certain band can be found from the inner product of coefficient array of the corresponding band with itself. The relationship between the energy $E_{\mathrm{MWP}}$ and 


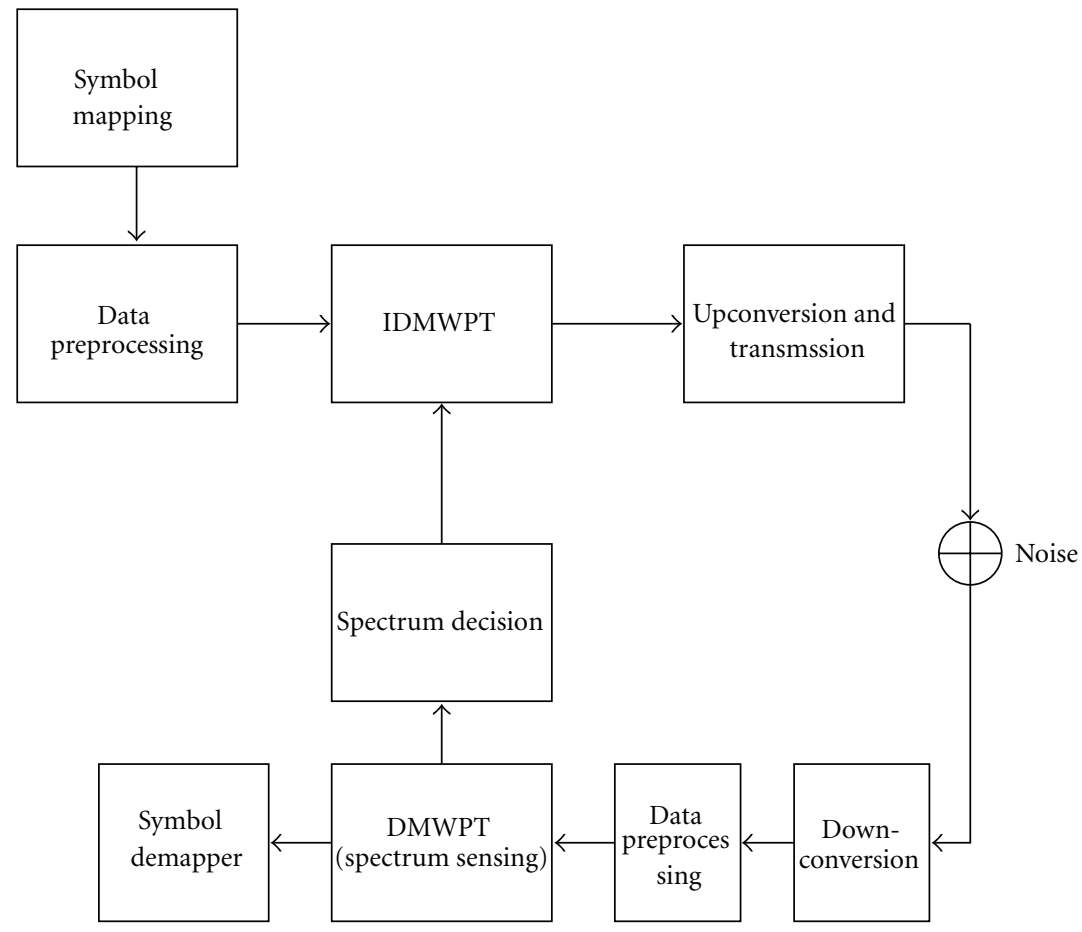

FIGURE 2: Multifilter based CR transceiver.

1: The iteration parameter $j$ is initialized to zero. The input signal is converted into a matrix of two rows by repeating the signal.

2: The low pass and high pass filter toeplitz matrices of the corresponding iteration are calculated.

3: The Multiwavelet packet coefficients are obtained.

4: Check whether the required iteration level is achieved. If yes go to next step. Otherwise increase the iteration parameter and repeat step 2 and 3.

5: If the required iteration level is reached the wavelet packet coefficients are saved and energy of the subbands are calculated. 6: The power spectral density is obtained and is compared with the predefined threshold.

7: Preliminary decision on vacant spectrum is taken based on step 6.

Algorithm 1

power $P_{\text {MWP }}$ for a node $m$ and total number of samples $K_{\text {sample }}$ is given as [26]

$$
P_{\mathrm{MWP}}^{(m)}=\frac{E_{\mathrm{MWP}}^{(m)}}{K_{\text {sample }}} .
$$

Power spectral density (PSD) of each subband can be calculated using the relation

$$
\operatorname{PSD}_{\mathrm{MWP}}^{(m)}=\frac{P_{\mathrm{MWP}}^{(m)}}{f_{\mathrm{MWP}}^{(m)}},
$$

where $f_{\text {MWP }}^{(m)}$ denotes the frequency range of a single subband. The PSD calculated for each subband can be compared with a predefined threshold to determine vacant bands. A simple algorithm for spectrum sensing using multiwavelets is given in Algorithm 1.

\section{MWPM in a Single-User CR Environment}

Multiwavelet filter banks can be used in both single-user and multiuser CR environments. As a preliminary work, the system model and signal model of a single-user CR transceiver are outlined in this section.

4.1. System Model. The CR transceiver for adaptive spectrum sharing using multiwavelet filter bank is shown in Figure 2. The system model is similar to scalar waveletbased CR transceiver with the replacement of multiwavelet packet transform instead of scalar wavelet transform. The vacant spectrum identification is done by measuring the power at the subbands of the demodulator output. That is, preliminary spectrum sensing is performed using discrete multiwavelet packet transform (DMWPT) as detailed in the previous section. Spectrum sensing requires vector input, and hence the signal stream under consideration is repeated 
based on multiplicity. Data preprocessing does signal repetition and serial-to-parallel conversion. The information from the preliminary spectrum sensing circuit is input to spectrum decision block. The spectrum decision block is a cross-layer approach which involves fine spectrum sensing at physical and MAC layers. Fine sensing at the physical layer incorporates feature extraction techniques to verify the presence of primary user (PU). Finally MAC layer takes the decision after considering certain link layer characteristics of the scanned frequency band and generates a binary information vector regarding spectrum occupancy. Once the free band is chosen by the spectrum decision block, the data symbols are mapped onto that spectrum. Similar to other transmultiplexers, inverse transform of multiwavelets is used for transmission. Due to the matrix structure of multifilters, multiwavelet-based transformation requires vectors instead of scalars. Preprocessing of data results in scalar-to-vector transformation and serial-to-parallel conversion. Inverse discrete multiwavelet transform (IDMWPT) is performed on the parallel data stream using vector extension of Mallat's algorithm by using transpose of the low-pass and highpass matrices $H$ and $G$. The process involved in IDMWPT block is shown in Figure 4. Each data symbol $\mathbf{X}[\mathbf{0}], \mathbf{X}[\mathbf{1}]$, and so forth are $r \times 1$ vector points where $r$ is the multiplicity [22]. $\mathbf{H}$ and $\mathbf{G}$ correspond to low-pass and high-pass multiwavelet filters. From Figure 4 it is evident that $r \cdot 2^{2}$ data symbols can be transformed into a serial stream by two levels of iteration. Successive iterations of upsampling and filtering operation can also be done by multiplication with corresponding toeplitz matrix. The details of single level multiwavelet packet transform and inverse transform using toeplitz matrix are discussed in [25]. The conditions to obtain error-free transmission under noiseless condition with multifilters are as follows.

(1) Each filter coefficient matrix should be a square matrix.

(2) For perfect reconstruction, the toeplitz matrix formed using filter coefficient matrices should be an orthogonal matrix.

At the receiver, the signal is downconverted and processed again to convert the serial stream into parallel vector symbols. DMWPT block demodulates the signal and then data is retrieved using symbol demapper.

4.2. The Signal Model. The signal transmitted from the CR node is given by

$$
\mathbf{s}_{\mathrm{MWP}}(k)=\frac{1}{\sqrt{r 2^{D}}} \sum_{i=1}^{r} \sum_{n=0}^{2^{D}-1} \mathbf{d}_{n, i} \Phi_{n, i}(k),
$$

where $\mathbf{d}$ is the vector representation of the data stream, $D$ is the number of iterations, $r$ is the multiplicity, and $\Phi_{n, i}(k)$ is the multiwavelet packet synthesis waveform. For ease of understanding, this expression can be represented with matrices. Let $\mathbf{X}$ denote the complete data matrix and $\mathrm{W}$ the transformation matrix or the corresponding toeplitz matrix. The signal transmitted from the node can now be written as

$$
\mathbf{s}_{\mathrm{MWP}}(k)=\mathbf{W}^{T}(k) \mathbf{X}(k),
$$

where $\mathbf{W}^{T}(k)$ is the transpose of the toeplitz matrix. At the receiver multiwavelet analysis is performed, and upon perfect synchronization and zero noise the demodulated signal is

$$
\tilde{\mathbf{s}}_{\mathrm{MWP}}(k)=\mathbf{W}(k) \mathbf{W}^{T}(k) \mathbf{X}(k),
$$

where $\tilde{\mathbf{s}}_{\mathrm{MWP}}(k)$ represents the demodulated signal component. Because of the orthonormality of the underlying multifilter banks, this can be simplified as

$$
\tilde{\mathbf{s}}_{\mathrm{MWP}}(k)=\mathbf{I}(k) \mathbf{X}(k),
$$

where $\mathbf{I}(k)$ is the identity matrix.

\section{MWP-MC-MA System for CR}

To exploit the unique features of multiwavelet filter banks within multi-user $\mathrm{CR}$ context, a new multicarrier and multiple access scheme called MWP-MC-MA is proposed in this work. It is the multiwavelet extension of WP-MC-MA proposed in [13]. The uplink and downlink system models are described in the subsequent subsections with necessary equations.

5.1. Uplink System Model. The basic uplink scheme of the proposed system is shown in Figure 3. The CR node scans its band of interest (BOI) and generates multiwavelet packet coefficient PSD vector (as described in the previous section) $C_{v}(n, i)$ where $n$ represents the subband index and $i$ is the corresponding multiwavelet base. Spectrum sensing requires vector input, and hence the signal stream under consideration is repeated based on multiplicity. Data preprocessing does signal repetition and serial-to-parallel conversion. It is evident that based on the number of wavelet functions (multiplicity) the scanned spectrum can be subdivided into a large number of uniform subbands. Based on the value of $C_{v}(n, i)$ and comparing it with predefined threshold, preliminary decision on vacant spectrum is taken. The information from the preliminary spectrum sensing circuit is input to spectrum decision block. The spectrum decision block is a cross layer approach which involves fine spectrum sensing at physical and MAC layers. Fine sensing at the physical layer incorporates feature extraction techniques to verify the presence of primary user (PU). Finally MAC layer takes the decision after considering certain link layer characteristics of the scanned frequency band and generates a binary information vector regarding spectrum occupancy. Spectrum allocation is made by modified-carrier assignment scheme (CAS) similar to that in [13]. The purpose of the CAS algorithm is to obtain the spectrum index vector $I_{m}$ and proper allocation of the subbands among CR users. The user's data are baseband encoded, preprocessed to obtain $r \times 1$ vector points instead of scalars, and channeled through $P$ parallel streams where $P$ is the number of subcarriers (subbands) allotted for each user. The data stream is fed into 
1: The binary spectrum information vector is obtained.

2: CAS tries to determine CSI of each free subband available within the BOI.

3: CSI of the CR node under consideration along with the neighboring nodes are obtained.

4: Based on the information obtained, CAS assigns subbands for the considered CR node. Two consecutive subbands will be allocated for the same user. The subbands with maximum SNR is the primary choice.

5: If the specified CR as well as a competing neighbor has maximum SNR at the $k$ th consecutive subbands, other CSI factors such as channel delay, phase offset will be taken into consideration to make the final decision.

6: Once the user and band is fixed, final decision is made and that subband index is included in the spectrum index vector $I_{m}$ and the $n$th vector data stream of the user is mapped onto those subbands.

7: The process is repeated until all the $P$ data streams of the user are mapped accordingly. The remaining subcarriers carry no data and are padded with zeros.

Algorithm 2

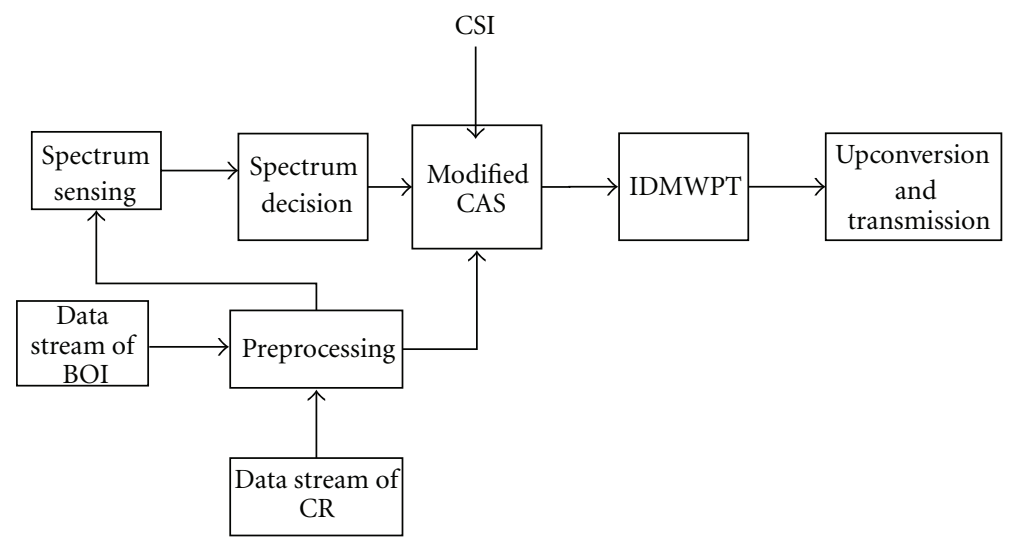

Figure 3: MWP-MC-MA uplink transmitter.

CAS, which will allocate subbands to the selected user based on the spectrum measurement vector $C_{v}(n, i)$ and CSI of the users within the given cell. The symbol stream is allocated to $P$ subbands within available $R$ subbands and by inserting $(R-P)$ zero arrays an $R$-dimensional vector is obtained as in OFDMA [38]. Mathematically it can be expressed as

$$
d_{n, i}^{(m)}= \begin{cases}c_{n, i}^{(m)} & \text { if } n, i \in I_{m} \\ 0 & \text { otherwise }\end{cases}
$$

where $c_{n, i}^{m}$ represents the constellation of encoded data stream of the user and $I_{m}$ is the set of the indices of $m$ th subchannel assigned to $m$ th user. The two-variable suffix of the data stream is due to multiwavelet bases and the additional subbands obtained due to them. The vector $d^{m}$ generated is input to the inverse discrete multiwavelet packet transform (IDMWPT) block for waveform modulation, and the serial data stream $s^{m}$ is obtained. Due to the time domain overlapping of multiwavelet bases, cyclic prefix (CP) is excluded.

5.1.1. Modified CAS of MWP-MC-MA System. The carrier allocation among different users is a difficult task in multifilter-based system compared to scalar wavelet-based system. This is due to the vector nature of data processing. When the multiplicity is two, each data vector should be a $2 \times 1$ matrix and each filtering in IDMWPT operates on two subbands of the available bandwidth. Hence for a transform size $N$, the

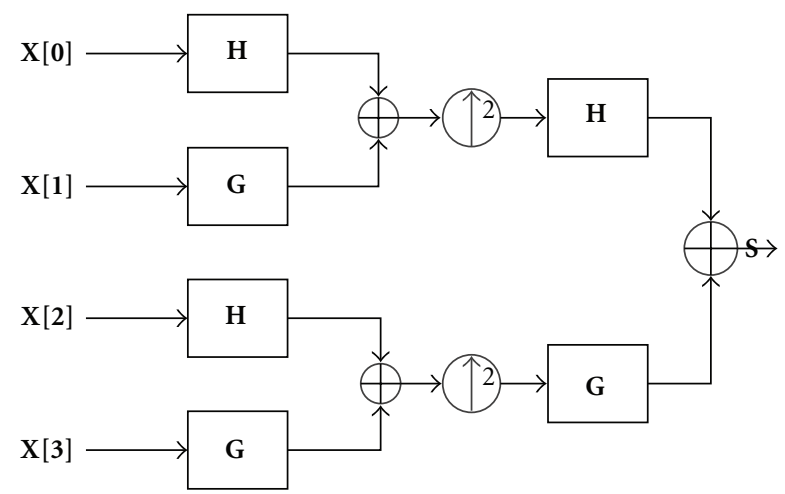

FIGURE 4: Inverse multiwavelet packet transform with two levels of iteration.

total available subbands will be $r \cdot 2^{N}$. However, dynamic allocation of subcarriers is possible only among $2^{N}$ bands. Modified CAS of multiwavelet-based system functions is described by Algorithm 2.

5.1.2. Data Preprocessing and IDWMPT. As mentioned in the spectrum sensing section, multiwavelet signal processing requires vectors instead of scalars. This is because convolution operation involved in wavelet synthesis (analysis) is to be performed with matrix filters. Hence the data stream of the 
CR node should also be converted into a vector format. Preprocessing of data performs scalar-to-vector transformation and serial-to-parallel conversion. To keep the block length of each MWPM symbol to be equivalent to that of WPM and OFDM, repeated row preprocessing methodology is not adopted and the available data stream is transformed to $r \times N / r$ vector datum where $N$ is the MWPM block length. IDMWPT is performed on the parallel data stream using vector extension of Mallat's algorithm by using transpose of the low-pass and high-pass matrices $H$ and $G$. The process involved in IDMWPT block is shown in Figure 4 and the process is similar to that explained in the single-user system model.

5.2. The Uplink Signal Model. The signal transmitted from the $m$ th CR node is

$$
s_{\mathrm{MWPm}}(k)=\frac{1}{\sqrt{r 2^{D}}} \sum_{i=1}^{r} \sum_{n=0}^{2^{D}-1} d_{n, i}^{m} \Phi_{n, i}(k)
$$

where $D$ is the decomposition level and $\Phi_{n, i}(k)$ corresponds to the multiwavelet synthesis waveform. The signal received at the base station is written as

$$
r(k)=\sum_{m=1}^{M} s_{\mathrm{MWPm}}^{\prime}(k) h_{m}(k)+n(k)
$$

where $n(k)$ is the noise and $s_{\mathrm{MWPm}}^{\prime}(k)$ is the multiwavelet packet modulated component of the $m$ th user and $h_{m}(k)$ the corresponding fading channel. The signal component can be written as

$$
s_{\text {MWPm }}^{\prime}(k)=e^{j \phi_{m}(k)} \sum_{n=0}^{R-1} \sum_{i=0}^{r} d_{n, i}^{m} \Phi_{n, i}^{\mathrm{syn}_{m}}\left(k-n R-\tau_{m}\right),
$$

where $d_{n, i}^{m}$ is the symbol stream mapped onto the subchannel of the $m$ th user as in (10) and $\Phi_{n, i}^{\mathrm{syn}_{m}}$ are multiwavelet packet synthesis waveforms for the $m$ th user subchannels. $R$ is the number subcarriers. The term $e^{j \phi_{m}}$ corresponds to the frequency and phase offset of the $m$ th user and is defined as

$$
\phi_{m}(k)=\frac{2 \pi \varepsilon_{m} k}{r R}+\theta_{m}(k),
$$

where $\theta_{m}$ is the phase noise component and $\varepsilon_{m}$ is the relative frequency offset of the $m$ th user. The product $r R$ gives the total number of subbands. The frequency offset is normalized to the inter carrier spacing. The integer timing offset $\tau_{m}$ is expressed in sampling periods. It evident that to maintain orthogonality among subcarriers during detection process, proper timing and frequency error estimation are required at the base station.

5.3. The Downlink System. The downlink transmitter and receiver are shown in Figures 5 and 6, respectively. After symbol mapping, the data stream of each user is divided into blocks. Similar to uplink, CAS unit maps the $P$ data symbols of each block onto subcarriers assigned to the corresponding user. The resultant vector will have data stream of all $M$ users summed up and fed to IDMWPT modulator and the data stream obtained serially is input to digital-to-analog Converter (D/A) and upconversion. At the receiver, the A/D output is the combination of data blocks of all users. Similar to the signal received at BS, frequency and timing errors are likely to be present. Hence the coarse frequency and timing estimation units are required to compute estimates of frequency and timing error. The frequency error estimate can be used to counter rotate the received sequence, and the timing error estimate is used for positioning of DMWPT window correctly. The channel equalization block corrects the channel impairments, if any, as well as the fractional timing errors. After the correction process, the data stream is input to DMWPT block where the serial stream is divided into subbands. For data detection, $P$ subbands of the particular user are considered from the available subbands.

5.4. Downlink Signal Model. The signal transmitted from the $\mathrm{BS}$ is

$$
\mathbf{s}_{\mathrm{MWP}}(k)=\frac{1}{\sqrt{r 2^{D}}} \sum_{i=1}^{r} \sum_{n=0}^{2^{D}-1} \mathbf{d}_{n, i} \Phi_{n, i}(k)
$$

where $\mathbf{d}$ denotes the vector representation of the summed data stream. The expression is similar to that of singleuser case, but $\mathbf{d}$ includes data of all $M$ users. By adopting matrix representation for data stream and multiwavelet modulation/demodulation, the signal transmitted from the BS can be written as

$$
\mathbf{s}_{\mathrm{MWP}}(k)=\mathbf{W}^{T}(k) \mathbf{X}(k),
$$

where $\mathbf{W}^{T}(k)$ is the transpose of the toeplitz matrix. Assuming AWGN channel at the receiver, the demodulated signal is

$$
\tilde{\mathbf{s}}_{\mathrm{MWP}}(k)=\mathbf{W}(k)\left(\mathbf{W}^{T}(k) \mathbf{X}(k)+\mathbf{N}(k)\right),
$$

where $\tilde{\mathbf{s}}_{\mathrm{MWP}}(k)$ represents the demodulated signal component. This can be elaborated as

$$
\tilde{\mathbf{s}}_{\text {MWP }}(k)=\left(\mathbf{W}(k) \mathbf{W}^{T}(k) \mathbf{X}(k)+\mathbf{W}(k) \mathbf{N}(k)\right) .
$$

Because of the orthonormality of the underlying multifilter banks, this can be simplified as

$$
\tilde{\mathbf{s}}_{\mathrm{MWP}}(k)=\mathbf{I}(k) \mathbf{X}(k)+\mathbf{W}(k) \mathbf{N}(k),
$$

where $\mathbf{I}(k)$ is the identity matrix. From the demodulated signal, the corresponding user's subbands can be extracted using the spectrum index vector and then data bits are obtained using symbol demapper.

\section{Performance Evaluation}

In this section the performance of the proposed system is analyzed in terms of spectrum measurement capability, error rate under different channel conditions, bandwidth efficiency, and computational complexity. 


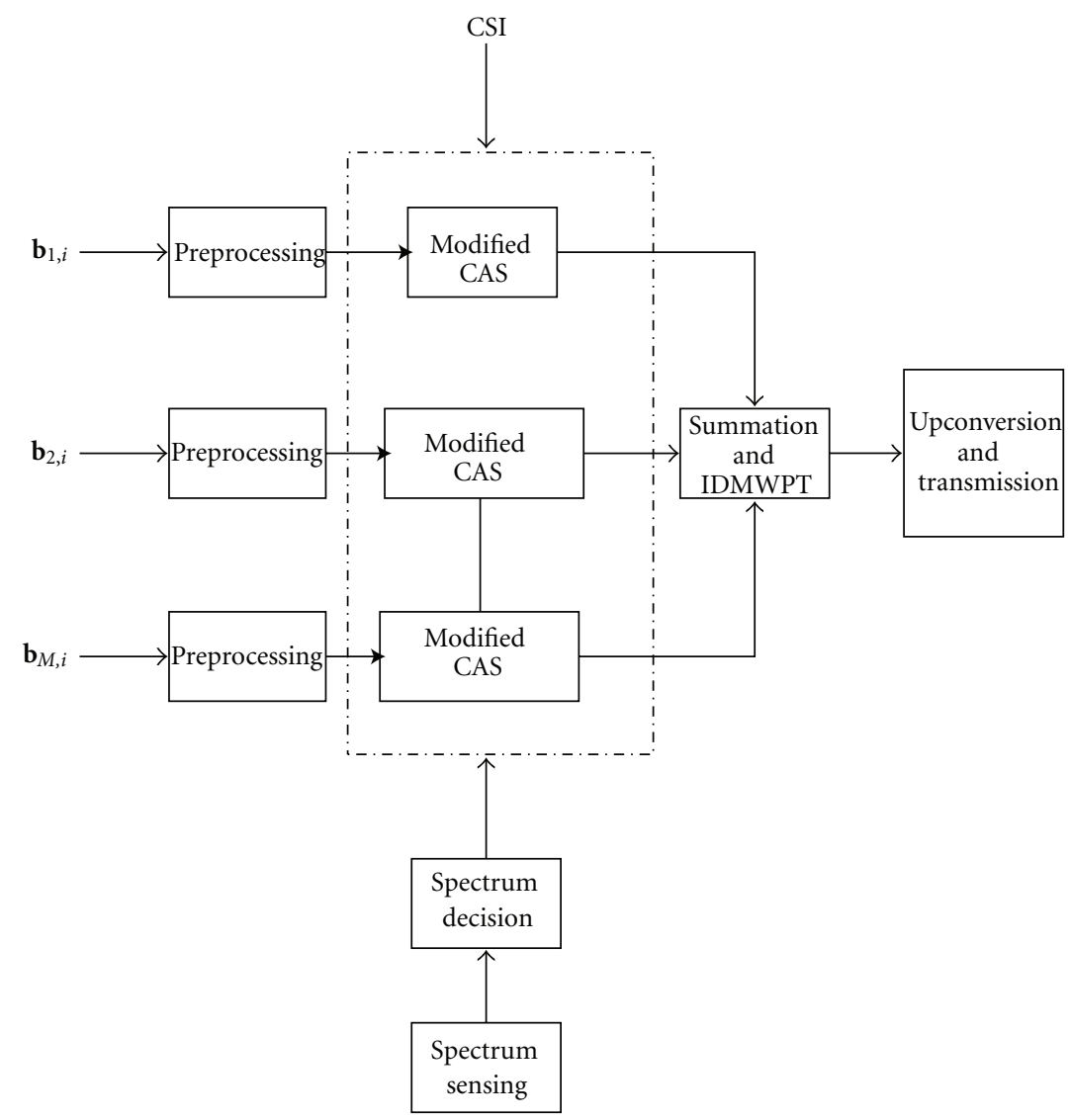

FIgURe 5: MWP-MC-MA downlink transmitter.

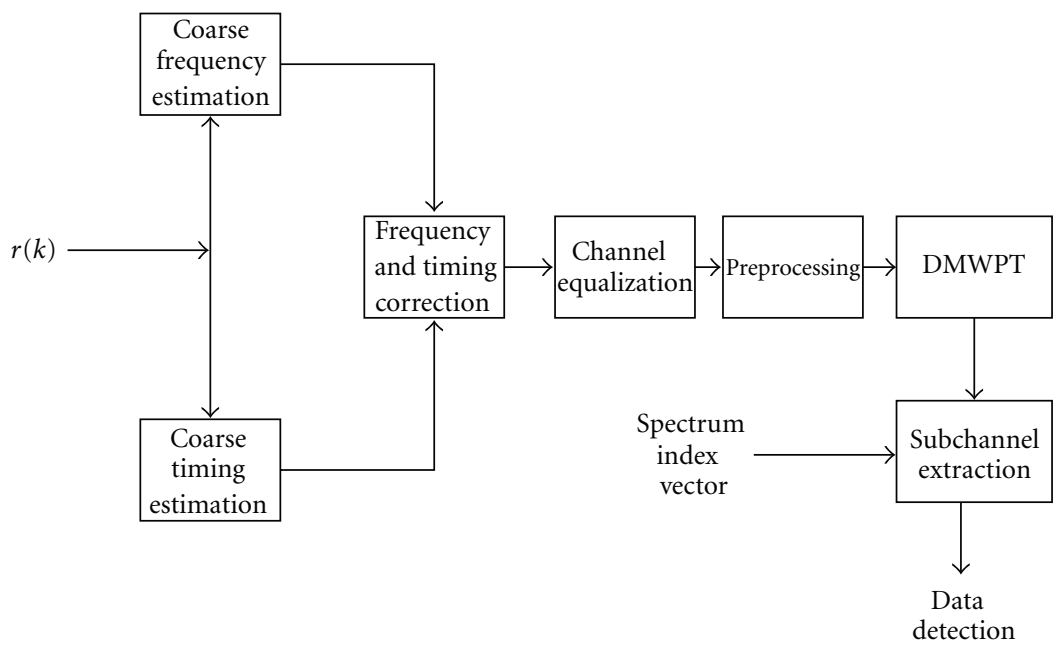

FIgURE 6: MWP-MC-MA downlink receiver.

6.1. Spectrum Measurements. A partial band BPSK signal with 100 symbols embedded in additive white Gaussian noise is considered as the signal to be detected. The carrier frequency is $300 \mathrm{MHz}$ and SNR is kept at $10 \mathrm{~dB}$. For simulation purpose, threshold is determined based on the principle of scalar wavelet-based detection technique explained in [12, 26]. In [12] the threshold for detecting vacant band is kept as $-7 \mathrm{~dB}$ as it gives probability of detection equal to 1 for a minimum probability of false alarm. The same threshold is adopted for multiwavelet-based system also due to the inherent similarity of both systems and for making a fair comparison. Figure 7 shows the useful signal detection using both symmetric multiwavelet and Symlet scalar wavelet. The given signal is decomposed into eight subbands with two 


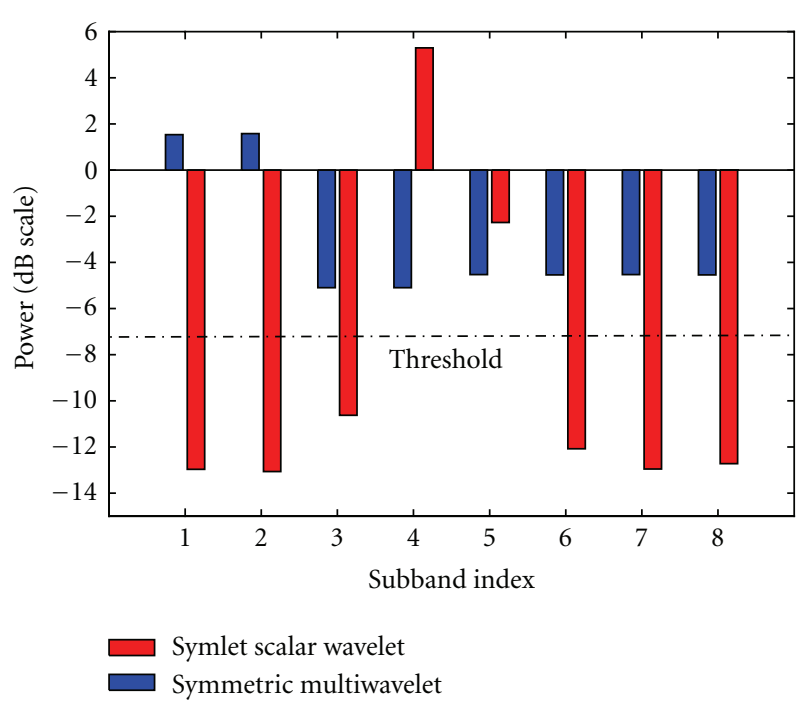

FIGURE 7: Spectrum sensing result of a partial band BPSK signal.

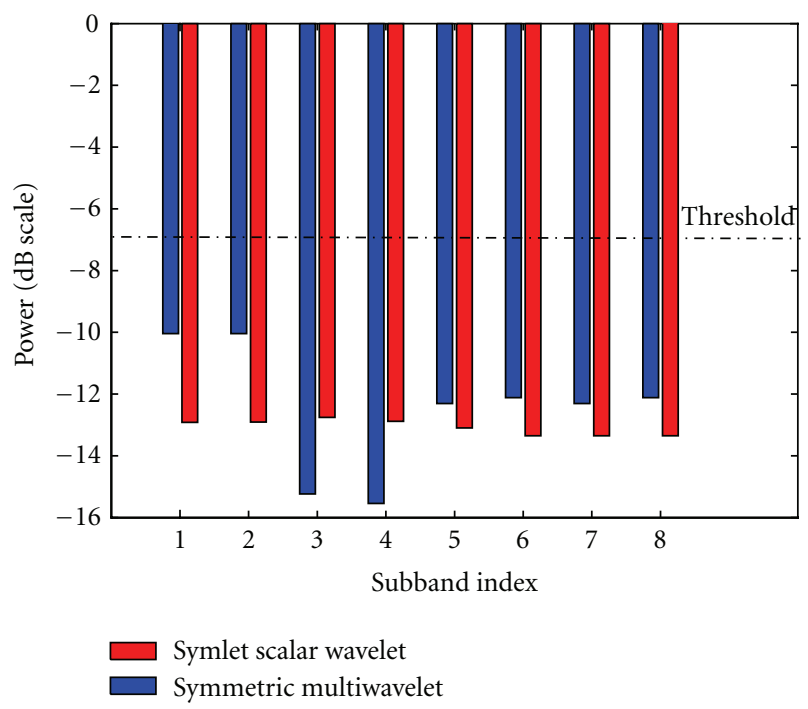

FIgURE 8: Spectrum sensing result of noise signal.

levels of iteration using multiwavelets where scalar wavelet requires three levels of iteration. The subband index is given on $x$ axis and power in $\mathrm{dB}$ is plotted on $y$ axis. It is evident that in the proposed system all subband power measurements are well above the threshold giving the presence of the signal. In the case of scalar wavelet, power measurements in only two subbands are above the threshold value. Hence it is proved that multiwavelet system is more powerful in detecting the presence of a primary user. Figure 8 shows the spectrum measurements corresponding to the noise signal detection. Both systems give accurate information about the free spectrum as subband power measurements are well below the threshold.

6.2. Error Performance under Different Channel Impairments. To evaluate the performance of the proposed system, a

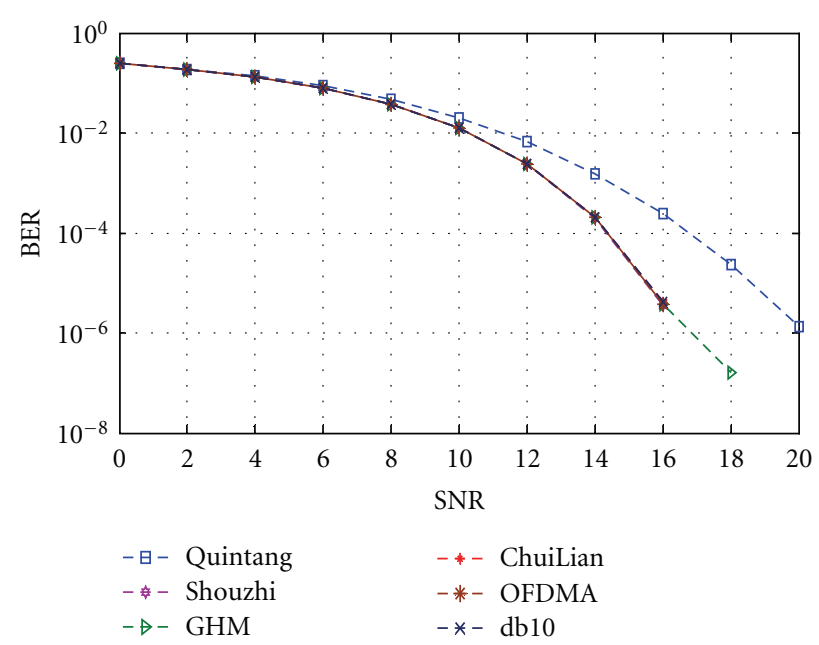

FIGURE 9: Error performance in flat fading channel.

multiuser CR environment with 4 users is considered. It is assumed that the BOI is scanned with multiwavelet packet transform and a total frequency band of 64 subbands found vacant. Equal number of subcarriers for all users is considered. Hence there are 4 subchannels with 16 subcarriers for each user in the simulated system. Actually this implies that each MWPM block corresponds to $64 / r$ multifilter subcarriers which handle $r \times 1$ vector data. The data of each user is converted into $r \times 1$ vector points and are given to IDMWPT block. It is assumed that there is perfect synchronization between users and base station (BS). The channel index set $I_{m}$ generated is known at the user end to separate the required data streams. The plots simulated in this work correspond to downlink channel of MWP-MCMA. It is assumed that QPSK modulation is the baseband symbol mapping scheme adopted by all users. Four different sets of multifilters discussed in [18-21] are used in the simulation and are named after the authors. The filter bank mentioned in [18] is termed as "GHM" (Gerenimo, Hardin, Massopust) and that in [19] as "ChuiLian" (Chui and Lian). The orthogonal multiwavelet with optimum time frequency resolution described in [20] is termed "Quintang" (Quintang Jiang) and the orthogonal filter bank described in [21] is denoted as "Shouzhi" (Yang Shouzhi). All multiwavelet families have multiplicity 2 and hence each filter coefficient is a $2 \times 2$ matrix. The number of filter coefficients and support length of each set are different and the details are given in [18-21]. The results obtained are compared with OFDMA and scalar WPM using Daubechies filter of length 20 (db10) with same number of users and subcarriers.

6.2.1. Error Performance in Single-Path Fading Channels. To incorporate the effects of flat fading, the channel gain is modeled as complex normal distribution $C N(0,1)$, with variance 1 . Figure 9 shows the BER in flat fading channel of different multicarrier schemes. It is assumed that the channel information is known at the receiver and is compensated. The different multiwavelets simulated in the work show similar performance except Quintang multiwavelet with 


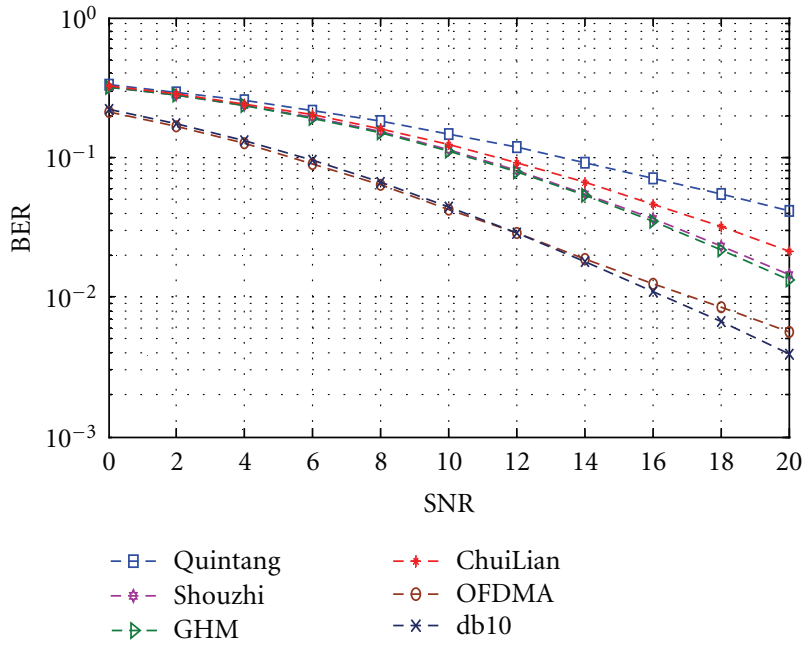

FIGURE 10: Error performance in single-path Rayleigh fading channel.

optimum time frequency resolution. It is evident that the BER of other multiwavelets are comparable with those of scalar wavelet-based system and OFDMA. In Figure 10 the effect of a single-path Rayleigh fading channel with a maximum Doppler shift of $55 \mathrm{rad} / \mathrm{sec}$ is plotted. The channel model is generated based on the Jakes model, and zero forcing equalization algorithm is used at the receiver. The proposed system is highly sensitive to the Doppler shifts compared to OFDMA and scalar WP-MC-MA.

6.2.2. Error Performance in Multipath Fading Channels. To evaluate the capability of the system to mitigate multipath fading channel impairments, three path fading channel with fixed and Rayleigh fading coefficients are considered. The cyclic prefix (CP) of the simulated OFDMA system is higher than the channel delay. At the receiver, the waveletbased scheme uses a time domain zero forcing equalizer with three taps per sample and OFDMA uses a frequency domain zero forcing equalizer with one tap per subcarrier since the demodulated OFDM signal is in the frequency domain. The fixed channel impulse response used for simulation is $h=\left[\begin{array}{lll}0.407 & 1 & 0.407\end{array}\right]$. Figure 11 shows the error performance under fixed fading multi-path and Figure 12 shows that under Rayleigh fading multi-path channel. When the channel coefficients are fixed the proposed multiwavelet based system using symmetric pair multiwavelets termed "Shouzhi" performs better than that of OFDMA and scalar wavelet system. Under multi-path Rayleigh fading channel condition zero-forcing equalizer fail to combat random changes in amplitude and phase, and hence the proposed system gives poor performance similar to scalar waveletbased system.

6.2.3. Effect of Phase Offset. As in any multicarrier modulation, it is important to consider the effect of frequency, phase and timing offset on error performance of the proposed system. Firstly the effect of phase noise on MWPM is considered. It is known that the presence of phase noise will

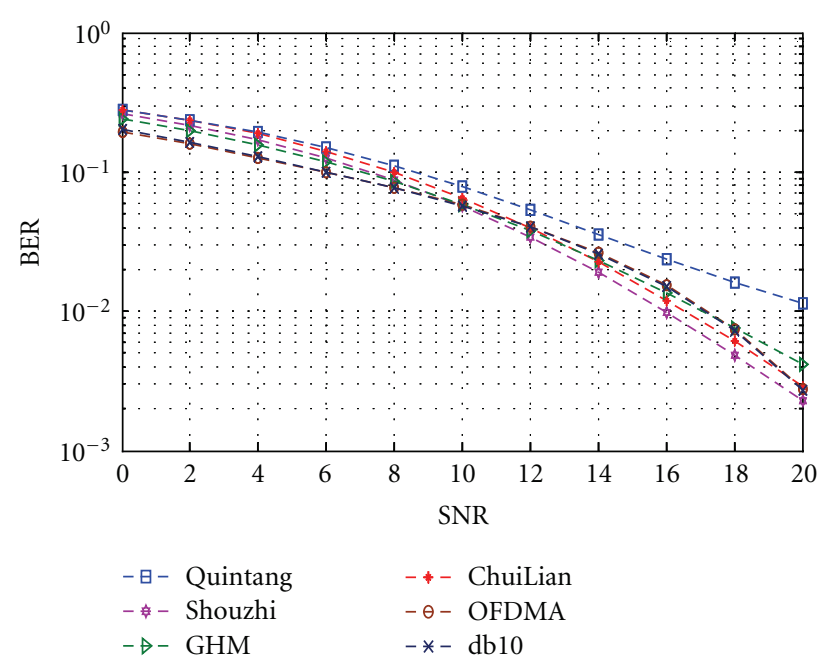

FIGURE 11: Error performance in multi-path channel with fixed fading coefficients.

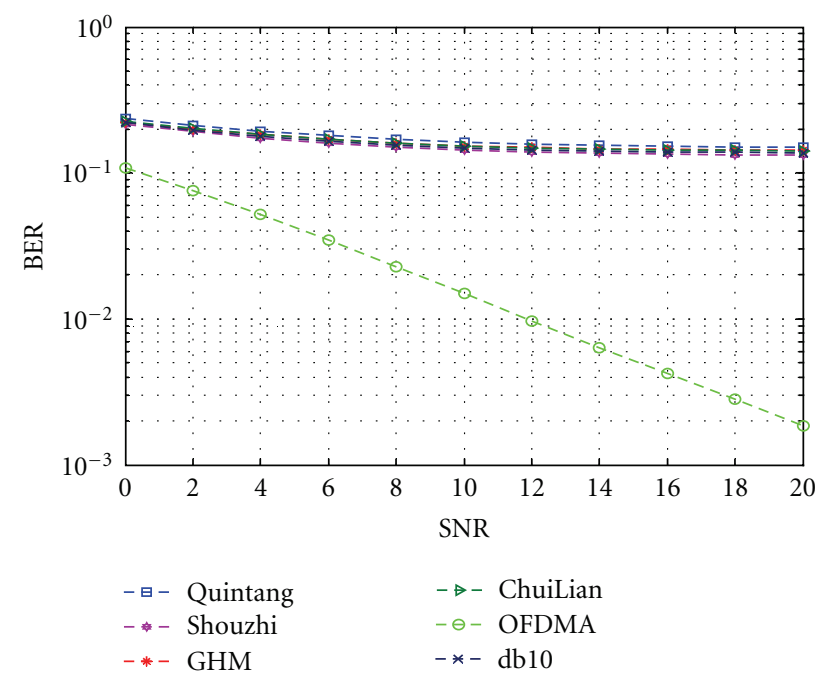

FIGURE 12: Error performance in multi-path channel with the Rayleigh fading coefficients.

affect the multicarrier modulation in two ways-(1) it will rotate all the constellation symbols by the same angle which is approximately equal to the average phase noise, (2) it will introduce inter-carrier interference (ICI) due to the spread of subcarriers with a larger bandwidth around the carrier frequency. To simulate the effects of phase noise in a multiuser environment, the phase noise is expressed as a zero mean Gaussian noise and the BER is calculated for different values of noise variance. The channel is assumed to be an AWGN channel and relative frequency error $\epsilon_{m}$ and timing offset to be zero. The signal received at the user terminal can be written as

$$
\mathbf{s}_{\mathrm{MWP}}(k)=\mathbf{W}^{T}(k) \mathbf{X}(k) e^{j \Theta(k)}+\mathbf{N}(k),
$$




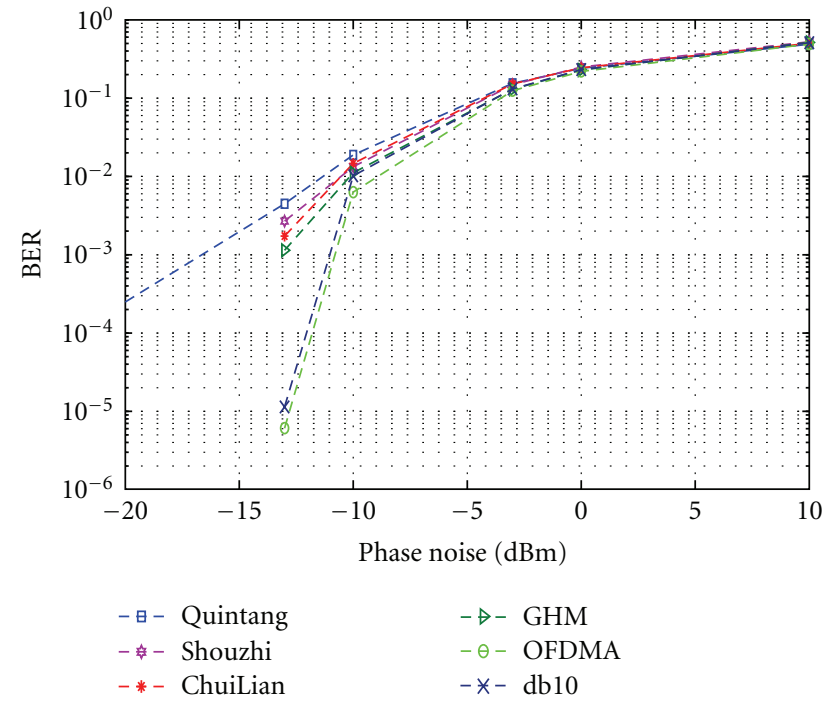

FIGURE 13: Effect of phase offset.

where $\Theta$ denotes the phase-offest vector and $\mathbf{N}(k)$ the noise vector. The demodulated signal would be

$$
\begin{aligned}
\tilde{\mathbf{s}}_{\mathrm{MWP}}(k) & =\mathbf{W}(k) \mathbf{W}^{T}(k) \mathbf{X}(k) e^{j \Theta(k)}+\mathbf{W}(k) \mathbf{N}(k) \\
& =\mathbf{I}(k) \mathbf{X}(k) e^{j \Theta(k)}+\mathbf{W}(k) \mathbf{N}(k) \\
& =\mathbf{X}(k) e^{j \Theta(k)}+\mathbf{W}(k) \mathbf{N}(k) \\
& =\frac{1}{\sqrt{r 2^{D}}} \sum_{i=1}^{r} \sum_{n=0}^{2^{D}-1} \mathbf{d}_{n, i} e^{j \Theta(k)}+\mathbf{W}(k) \mathbf{N}(k) .
\end{aligned}
$$

It is evident that the signal constellations are rotated by the corresponding phase angle and the noise component is altered by the respective multifilter coefficients. Figure 13 shows the effect of phase offset on error performance when the signal-to-noise ratio is kept constant at $15 \mathrm{~dB}$. The phase noise variance is expressed in $\mathrm{dB}_{m}$ and is given on $x$ axis. The bit error rate is depicted on $y$ axis. Figure shows that error increases considerably as the phase noise approaches zero and the type of multifilter does not improve the performance. OFDMA and scalar WPM perform comparatively better than multifilter-bank-based system.

6.2.4. Effect of Frequency Offset. Frequency offset can occur due to the Doppler shift or by misalignment between the transmitted carrier frequency and the locally generated carrier frequency at the receiver. It destroys orthogonality among subcarriers and hence causes severe ICI. To simulate the effect of frequency offset, AWGN channel is considered and the phase and timing offsets are assumed to be zero. Figure 14 shows the effect of frequency error on performance. BER is plotted as a function of relative frequency offset when the SNR is kept constant as $15 \mathrm{~dB}$. No error compensation is done at the receiver. Other than "Quintang" multifilter, all other systems have comparable error performance. The error degradation of Quintangbased systems is due to optimization of its time frequency resolution.

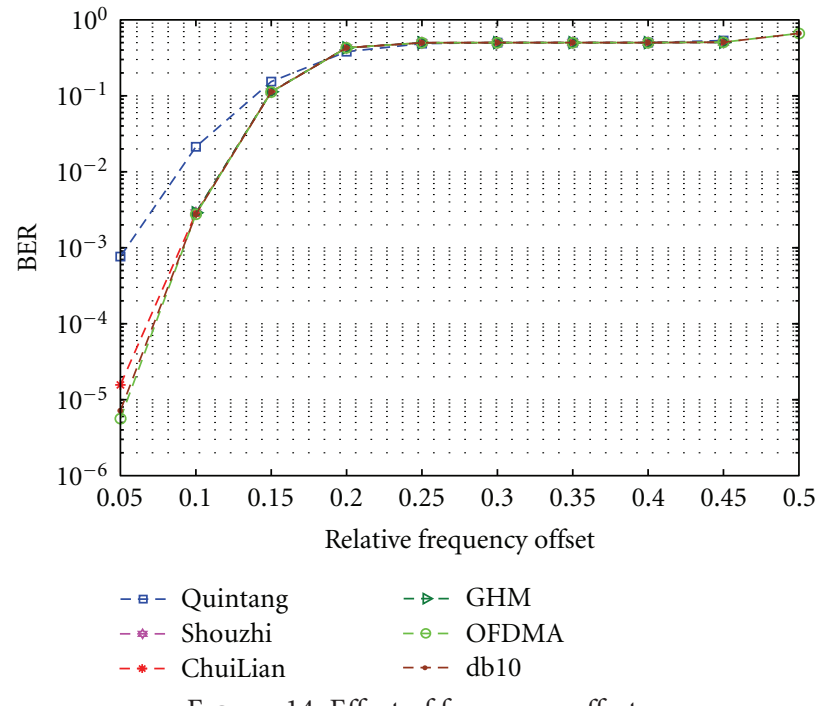

FIgURE 14: Effect of frequency offset.

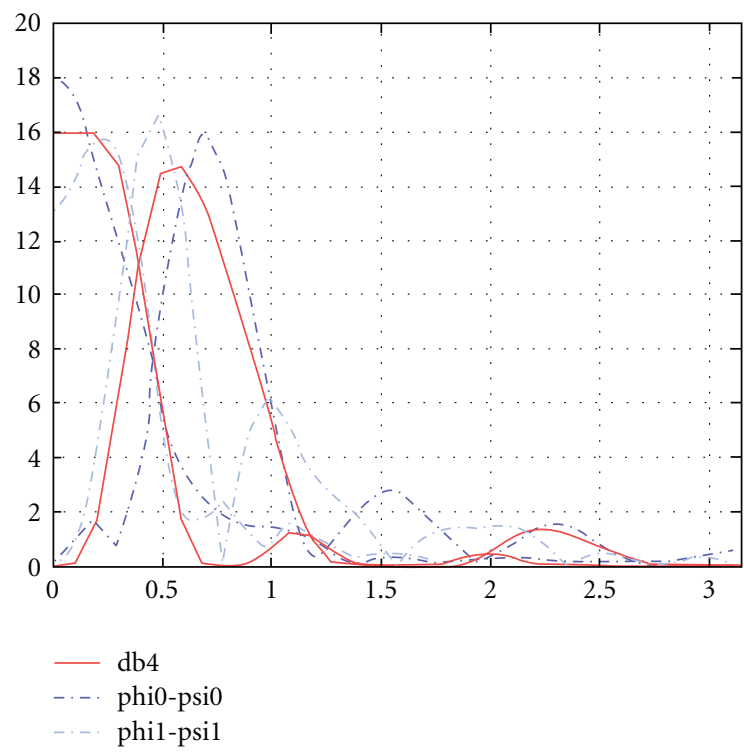

Figure 15: Spectrum of "GHM" and the Daubechies wavelets.

6.3. Bandwidth Efficiency and Sidelobe Suppression. Due to matrix-structured filter bank, multiwavelet-based systems possess higher bandwidth efficiency compared to those of scalar wavelet and OFDMA system. In the proposed system multifilters of multiplicity two are considered. Hence there can be twice the number of subbands compared to those of OFDM and WPM for a given iteration. The spectrum of multiwavelet of multiplicity two and Daubechies scalar wavelet are illustrated in Figure 15 [22]. It is evident that there are only two subcarriers for scalar wavelet (red curves) but four subcarriers for multiwavelet system (blue dotted curves) for a given bandwidth. Thus bandwidth efficiency will be twice that of scalar WPM and OFDM. Moreover similar to scalar WPM, multiwavelet system does not include $\mathrm{CP}$ along with the signal, and hence the spectral efficiency will be always higher than that of OFDM. Lastly the spectral 
TABLE 1: Complexity analysis.

\begin{tabular}{lccc}
\hline Name & Filter length & $\begin{array}{c}\text { Number of } \\
\text { subbands }\end{array}$ & $\begin{array}{c}\text { Number of } \\
\text { multiplications }\end{array}$ \\
\hline Quintang & 7 & 128 & 1792 \\
Shouzhi & 3 & 128 & 768 \\
GHM & 4 & 128 & 1024 \\
ChuiLian & 4 & 128 & 1024 \\
Daubechies & 20 & 64 & 2560 \\
OFDM & - & 64 & 1024 \\
\hline
\end{tabular}

leakage is also minimum in multiwavelet-based system due to nonrectangular waveforms in time domain.

6.4. Complexity Analysis. Conceptually, multiwavelet packet transform suffers from higher complexity involved in each level of its computation. When the filter coefficients are $2 \times 2$ matrices, the computations required in one level of iteration will be approximately twice to that of scalar wavelet transform. But as multiwavelet transform can provide twice the number of subbands in a given iteration compared to scalar WPM, it is expected that for a fixed number of subbands, the computational complexity would be almost similar. Theoretically, for an N stage WPT, the number of real multiplications required to calculate the scalar wavelet coefficients is always less than $2 L T$ where $T=2^{N}$ and $L$ is the filter length [39]. In the case of multiwavelets, this expression can be approximated as $r \cdot 2 L T$ since the additional computations in each level of the transform depends on multiplicity. The total number of real multiplications required for different orthonormal multiwavelet systems, Daubechies wavelet (db10), and OFDM for transform stage $N=6$ is given in Table 1. It is shown that due to lower number of filter taps, "Shouzhi," "GHM," and "ChuiLian" multiwavelet based systems have computational complexity comparable or lower than that of OFDM for a given $N$.

\section{Conclusion and Future Work}

In this work, a novel multicarrier multiple-access scheme for $\mathrm{CR}$ using multiwavelet packet modulation has been proposed. The MWP-MC-MA system provides spectrum efficiency, flexibility, orthogonality, and multi-user support. A new spectrum sensing method using multifilter bank is proposed. Modified CAS unit has been exploited for adaptive subcarrier allocation. The simulation results show that proposed system insures good flexibility, spectrum efficiency, and comparable error performance. Preliminary spectrum measurement is also possible using DMWPT, and computational complexity is moderate due to lower number of multifilter coefficients. Future work will involve the performance analysis of the multifilter-based spectrum sensing and flexibility enhancement of the proposed system by enabling noncontiguous band operation.

\section{References}

[1] S. Haykin, "Cognitive radio: brain-empowered wireless communications," IEEE Journal on Selected Areas in Communications, vol. 23, no. 2, pp. 201-220, 2005.

[2] T. A. Weiss and F. K. Jondral, "Spectrum pooling: an innovative strategy for the enhancement of spectrum efficiency," IEEE Communications Magazine, vol. 42, no. 3, pp. S8-S14, 2004.

[3] I. F. Akyildiz, W. Y. Lee, M. C. Vuran, and S. Mohanty, "NeXt generation/dynamic spectrum access/cognitive radio wireless networks: a survey," Computer Networks, vol. 50, no. 13, pp. 2127-2159, 2006.

[4] C. Carlos, K. Challapali, D. Birru, and S. Shankar, "IEEE 802.22: an introduction to the first wireless standard based on cognitive radios," Journal on Communications, vol. 1, no. 1, 2006.

[5] J. Y. Won, S. B. Shim, Y. H. Kim, S. H. Hwang, M. S. Song, and C. J. Kim, "An adaptive OFDMA platform for IEEE 802.22 based on cognitive radio," in Proceedings of the Asia-Pacific Conference on Communications (APCC '06), August 2006.

[6] A. M. Wygliski, M. Nekovee, and Y. T. Hou, Cognitive Radio Communications and Networks: Principle and Practice, Academic Press, 2010.

[7] A. Jamin and P. Mähönen, "Wavelet packet modulation for wireless communications," Wireless Communications and Mobile Computing, vol. 5, no. 2, pp. 123-137, 2005.

[8] M. K. Lakshmanan, I. Budiarjo, and H. Nikookar, "Maximally frequency selective wavelet packets based multi-carrier modulation scheme for cognitive radio systems," in Proceedings of the 50th Annual IEEE Global Telecommunications Conference (GLOBECOM '07), pp. 4185-4189, November 2007.

[9] D. Karamehmedović, M. K. Lakshmanan, and H. Nikookar, "Performance evaluation of WPMCM with carrier frequency offset and phase noise," Journal of Communications, vol. 4, no. 7, pp. 496-508, 2009.

[10] M. Baro and J. Ilow, "Multi-band wavelet-based spectrum agile communications for cognitive radio secondary user communication," in Proceedings of the IEEE International Symposium on Broadband Multimedia Systems and Broadcasting, Broadband Multimedia Symposium (BMSB '08), March 2008.

[11] H. Nikookar and M. K. Lakshmanan, "Comparison of sensitivity of OFDM and wavelet packet modulation to time synchronization error," in Proceedings of the IEEE 19th International Symposium on Personal, Indoor and Mobile Radio Communications (PIMRC '08), September 2008.

[12] M. K. Lakshmanan, D. D. Ariananda, and H. Nikookar, "Cognitive radio transmission and spectrum sensing using a wavelet packet transceiver," in Proceedings of the IEEE 20th Personal, Indoor and Mobile Radio Communications Symposium, (PIMRC '09), September 2009.

[13] M. Mathew, A. B. Premkumar, and C. T. Lau, "Multiple access scheme for multi user Cognitive Radio based on wavelet transforms," in Proceedings of the IEEE 71st Vehicular Technology Conference (VTC'10), May 2010.

[14] V. Strela, Multiwavelets: theory and applications [Ph.D. thesis], Massachusetts Institute of Technology, 1996.

[15] V. Strela, P. N. Heller, G. Strang, P. Topiwala, and C. Heil, "The application of multiwavelet filterbanks to image processing," IEEE Transactions on Image Processing, vol. 8, no. 4, pp. 548$563,1999$.

[16] B. Behera, "Multiwavelet packets and frame packets of L2 ( $\mathbb{R d}$ )," Proceedings of the Indian Academy of Sciences, vol. 111, no. 4, pp. 439-463, 2001. 
[17] G. Strang and V. Strela, "Short wavelets and matrix dilation equations," IEEE Transactions on Signal Processing, vol. 43, no. 1, pp. 108-115, 1995.

[18] J. S. Geronimo, D. P. Hardin, and P. R. Massopust, "Fractal functions and wavelet expansions based on several scaling functions," Journal of Approximation Theory, vol. 78, no. 3, pp. 373-401, 1994.

[19] C. Chui and J. Lian, A Study of Orthonormal Multiwavelets, Centre for Approximation Theory, 1995.

[20] Q. T. Jiang, "On the design of multifilter banks and orthonormal multiwavelet bases," IEEE Transactions on Signal Processing, vol. 46, no. 12, pp. 3292-3303, 1998.

[21] Y. Shouzhi, "A fast algorithm for constructing orthogonal multiwavelets," ANZIAM Journal, vol. 46, no. 2, pp. 185-201, 2004.

[22] M. L. You and J. Ilow, "An application of multi-wavelet packets in digital communications," in Proceedings of the 2nd Annual Conference on Communication Networks and Services Research, pp. 10-18, May 2004.

[23] M. You and J. Ilow, "Adaptive multi-wavelet packet modulation for channels with time-frequency localized interference," in Proceedings of the IEEE 6th Circuits and Systems Symposium on Emerging Technologies: Frontiers of Mobile and Wireless Communication, pp. 441-444, June 2004.

[24] X. H. Yan and G. Z. Liu, "Multiwavelet packet based ofdm system," in Proceedings of the IEEE Asia-Pacific Conference on Circuits and Systems, SoC Design for Ubiquitous Information Technology (APCCAS '04), pp. 681-684.

[25] A. H. Kattoush, W. A. Mahmoud, and S. Nihad, "The performance of multiwavelets based OFDM system under different channel conditions," Digital Signal Processing, vol. 20, no. 2, pp. 472-482, 2010.

[26] D. D. Ariananda, M. K. Lakshmanan, and H. Nikookar, "A study on application of wavelets and filter banks for cognitive radio spectrum estimation," in Proceedings of the 2nd European Wireless Technology Conference (EuWIT '09), pp. 218-221, September 2009.

[27] D. Cabric, S. M. Mishra, and R. W. Brodersen, "Implementation issues in spectrum sensing for cognitive radios," in Conference Record of the 38th Asilomar Conference on Signals, Systems and Computers, pp. 772-776, November 2004.

[28] T. Yücek and H. Arslan, "A survey of spectrum sensing algorithms for cognitive radio applications," IEEE Communications Surveys and Tutorials, vol. 11, no. 1, pp. 116-130, 2009.

[29] B. Farhang-Boroujeny, "Filter bank spectrum sensing for cognitive radios," IEEE Transactions on Signal Processing, vol. 56, no. 5, pp. 1801-1811, 2008.

[30] Y. Youngwoo, J. Hyoungsuk, J. Hoiyoon, and L. Hyuckjae, "Discrete wavelet packet transform based energy detector for cognitive radios," in Proceedings of the IEEE 65th Vehicular Technology Conference (VTC '07), pp. 2641-2645, April 2007.

[31] Y. Hur, J. Park, W. Woo et al., "A wideband analog MultiResolution Spectrum Sensing (MRSS) technique for cognitive radio (CR) systems," in Proceedings of the IEEE International Symposium on Circuits and Systems (ISCAS '06), pp. 40904093, May 2006.

[32] L. C. Wang, C. W. Wang, Y. C. Lu, and C. M. Liu, "A concurrent transmission MAC protocol for enhancing throughout and avoiding spectrum sensing in cognitive radio," in Proceedings of the IEEE Wireless Communications and Networking Conference (WCNC '07), pp. 121-126, Hong Kong, China, March 2007.

[33] Y. Zeng and Y. C. Liang, "Maximum-minimum eigenvalue detection for cognitive radio," in Proceedings of the 18th Annual
IEEE International Symposium on Personal, Indoor and Mobile Radio Communications (PIMRC '07), September 2007.

[34] Z. Lei and F. Chin, "A reliable and power efficient beacon structure for cognitive radio systems," in Proceedings of the IEEE International Conference on Communications (ICC '08), pp. 2038-2042, May 2008.

[35] H. Arslan and T. Yucek, "Spectrum sensing for cognitive radio applications,", in Cognitive Radio, Software Defined Radio, and AdaptiveWireless Systems, H. Arslan, Ed., Springer, New York, NY, USA, 2007.

[36] Y. Zeng and Y. C. Liang, "Eigenvalue-based spectrum sensing algorithms for cognitive radio," IEEE Transactions on Communications, vol. 57, no. 6, pp. 1784-1793, 2009.

[37] Z. Quan, S. J. Shellhammer, W. Zhang, and A. H. Sayed, "Spectrum sensing by cognitive radios at very low SNR," in Proceedings of the IEEE Global Telecommunications Conference (GLOBECOM '09), pp. 1-6, November 2009.

[38] M. Morelli, C. C. J. Kuo, and M. O. Pun, "Synchronization techniques for orthogonal frequency division multiple access (OFDMA): a tutorial review," Proceedings of the IEEE, vol. 95, no. 7, pp. 1394-1427, 2007.

[39] G. Strang and T. Nguyen, Wavelets and Filter Banks, Wellesley Cambridge Press, 1996. 

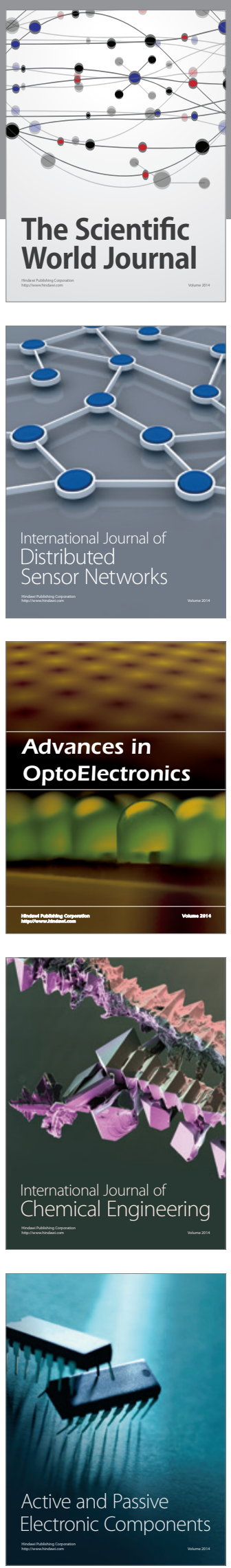
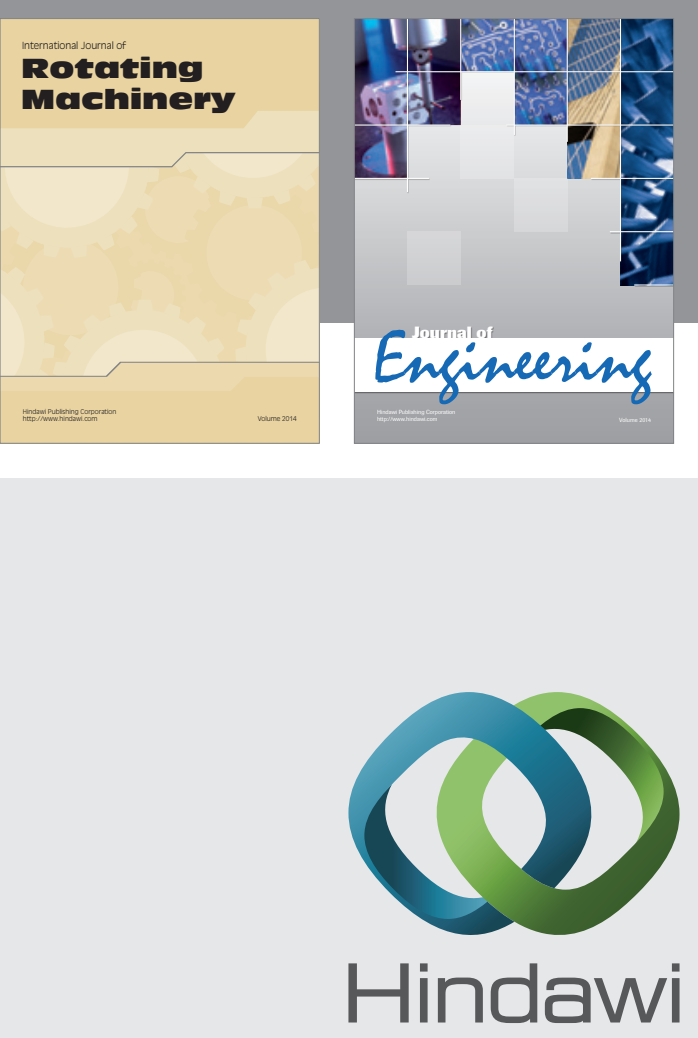

Submit your manuscripts at

http://www.hindawi.com
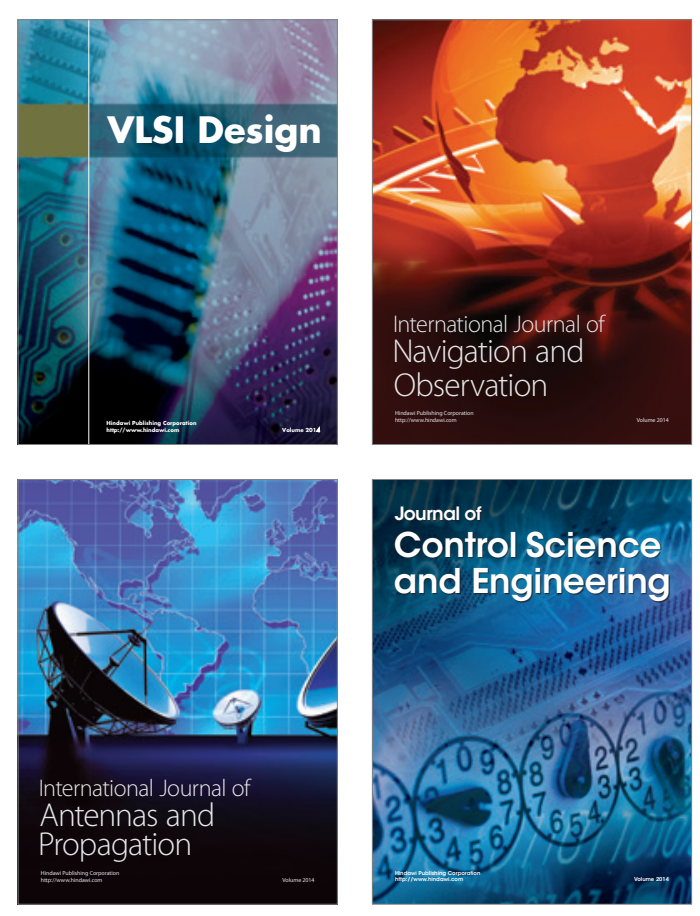
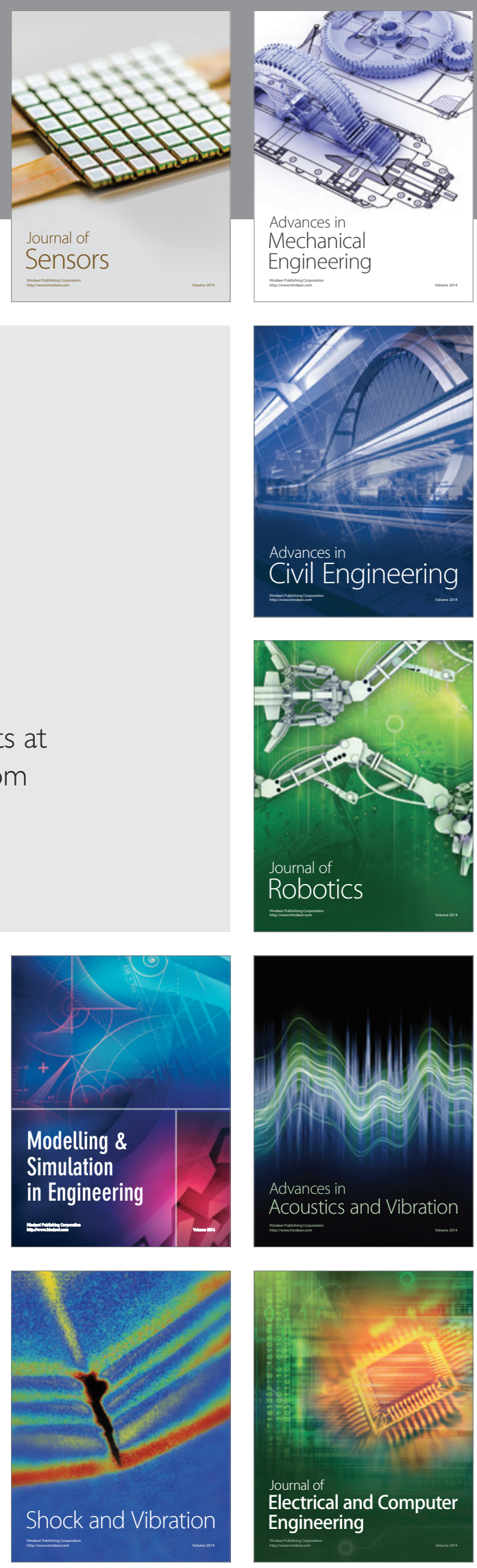